\title{
EDITORIAL
}

\section{Get the message out!}

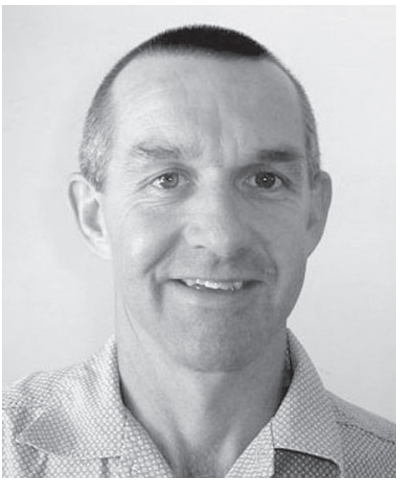

Every couple of months an incident in the sporting world triggers a passionate public debate. Members of the public are quick to form and express their opinion. Often the area of discussion is beyond the scope of expertise of the person contributing to the debate; however this does not deter them from doing so with gusto. The media often get involved in these debates and seek out anyone who will give them a dial-a-quote on the incident. The most recent example which springs to mind is the discussion about 'Wayne Rooney's metatarsal'. Soon after his injury everyone from the managing director of a clothing outlet to a shoe shiner at the airport became an expert about 'metatarsals' and had an opinion about whether or not Rooney would make it to the World Cup. Most often the opinions of the public in these specialist areas are shaped by the media, be it coaching strategies or medical problems. When covering one of these topics a responsible media reporter seeks out authoritative people with expertise in the area who are able to give an evidence-based opinion. The less responsible media seek out anyone for a 'dial-a-quote'; the result - unclear or misinformation which does little to educate the public.

A relatively recent trend which compounds the problem of perpetuating myths and unclear information is the growth of blogs, or discussion sites on the Internet. The ready access to blog sites makes anyone who chooses an instant selfproclaimed expert. One only has to read the rugby websites to see the strong opinions expressed by some bloggers about coaching style, player fatigue and treatment of injuries. While the public debates on these sites are sometimes informative and amusing it is also the platform for the expression of radical views. It seems to be that views which are expressed loudly and frequently, shift from being radical views and assume a more central stage.

Where is all this leading? The point I am trying to make is that as a health professional, one shares a growing responsibility to get factual, evidenced-based and practical advice into circulation so that the end users, most often the public, have access to it. When a pseudo-scientist says that eating a certain supplement on a daily basis will make muscles grow faster than anabolic steroids, challenge the statement! When a 'wannabe sports physician' makes a public statement about player $X$ being able to play a match with a broken bone, challenge it! The public is hungry for factual information on matters in which members of the South African Sports Medicine Association are experts. Use whatever platform you can to get the information out to the public. Make sure the information is accurate and practical. Only then will the public be well informed.

This edition of the Journal has two original research papers, one review and one short communication. The first study, by Tanja Oosthuyse and Andrew Bosch, examined the effects of various stages of the menstrual cycle on ventilatory responses during submaximal exercise. They showed that there was indeed a relationship between ovarian hormone concentration and ventilatory responses. The authors made the point that these findings have a practical relevance for research design. The next study by Justin Durandt and colleagues profiled the top under- 16 and under- 18 rugby players in the country. While there were some logical and expected differences between groups and playing positions for body composition and measurements of fitness, it was surprising to note that sprinting speed was similar between the under-16 and under-18 players. The data from this study can be used for comparative purposes, or for talent identification in the various playing positions. The review study by Andrew McKune examined the recent research on asthma, airway hyper-responsiveness and airway inflammatory responses in athletes. This is a comprehensive review and provides an excellent update. The short communication by Adrian Kilian and Richard Stretch reported on the number of medical cases for spectators at the 2003 Cricket World cup, including the opening ceremony. The data in this paper are useful for administrators and medical support staff involved in the planning of future sports events.

I hope you enjoy this second 2006 edition of the South African Journal of Sports Medicine!

\section{Mike Lambert}

Editor-in-Chief 